\title{
The Effect and Prospect of the Joining of Linguistic Theory and Chinese Teaching
}

\author{
Jiugen Xiao, Mengwei Huang
}

Jiangxi Normal University Language and Language Life Research Center, Nanchang, China.

*Jiugen Xiao

Keywords: Linguistic theory; Chinese teaching; joint; Role; Prospects

\begin{abstract}
Linguistic theory is the foundation of Chinese teaching, while Chinese teaching is the practice of linguistic theory, both of which have close relationship. In recent years, though the linguistic theory and Chinese teaching practice has jointed together to a certain degree, we still need to advance towards more wide fields and do further studies in order to achieve the complete joint between the two. The practice has proved that once the linguistic theory has been successfully jointed with the Chinese teaching, it will not only bring positive significance to Chinese teaching, but also bring more prospects to the construction of Chinese teaching.
\end{abstract}

\section{语言学理论与语文教学实现成功对接的作用和前景}

\author{
肖九根 黄梦伟 \\ 江西师范大学 语言与语言生活中心, 南昌, 中国 \\ *肖九根
}

关键词：语言学理论；语文教学；对接；作用；前景

摘 要: 语言学理论是语文教学的基础, 而语文教学又是语言学理论的实践活动, 二者关系十 分密切。近些年来, 尽管语言学理论与语文教学实践已有一定程度的对接, 但要真正实现二 者的无缝对接, 还需向更深广的领域推进, 还要做更深入细致的研究工作。实践证明, 语言 学理论一旦同语文教学实践实现了成功对接, 这不仅对语文教学目标的实现会产生积极意义, 而且还会给语文教学学科建设带来更为广阔的前景。

1. 引言

长期以来，我国语言研究基本上是以语言本体为主，而不太关注语文教学问题或者关注 得甚少, 尤其是没有从基础语文教学实践需要出发去开展高师语言教学与语言研究, 致使语 言学理论没有为基础语文教学实践提供有效的指导, 而传统语言学理论有些较为繁琐且不易 理解, 也未随着时代的发展而满足适应其实际需要的根本要求。这样一来, 基础语文教育就 淡化了语言学知识理论, 以致长期脱离语言学基础理论的指导, 这就形成了一个恶性循环, 难以真正实现二者的有效对接。近些年来, 应用语言学研究悄然兴起, 但是对基础语文教学 而言, 将其理论与之结合起来研究的, 还是少之又少。本来, 语文教学是传统语言学重要的 应用领域, 语文教学的科学品位在很大程度上取决于语言研究的科学品位。要真正解决这一 问题, 一方面语言学研究要切实重视语文教育问题, 为语文学科提供行之有效的动态言语知 识理论体系, 另一方面基础语文教育也要深入思考如何将动态言语知识理论体系转化为语文 
教育范畴的言语知识理论基础, 从而实现语文学科与语言知识理论的真正融合, 而不再是简 单的抵触或一味地淡化语言知识理论。

\section{2. 语言学理论与语文教学实践实现成功对接的重要作用}

\section{1 语言学理论在语文教学实践中对学生的智能培养具有十分重要的作用}

语言学理论告诉我们: 语言不是本来就有的, 它是随着人类的社会实践活动逐渐形成和 发展起来的。语言既是一种社会现象, 是社会的一种交际工具, 同时也是一种心理现象, 是 人类的一种思维工具。思维功能是语言功能的另一重要方面, [1](第10页)是人的智能活动必 不可少的要素, 是人们摄取外部信息进行加工不可或缺的手段。思维能力是我们智能的核心 部分。传统意义上的思维主要是从哲学、逻辑学角度定义的。我们应该认识思想与思维的不 同, 这样才便于将语言学理论运用到基础语文教学实践中去。思想是人们对现实世界的认识, 而思维是认识现实世界的动脑筋过程, 同时也是比较、分析、综合认识现实的一种能力。[1](第 10页)另外, 思维是依靠语言符号进行的, 思维的结果即思想是靠语言符号的物化而固定而表 现的。[2](第362页)思维可以分成形象思维、动作思维、抽象思维等多种类型。形象思维是最 基本的艺术思维形式, 形象思维以表象为基础。抽象思维是一种理性的、逻辑的、分析的思 维形式, 抽象思维以概念为基础。生动的表象和抽象的概念成为人类思维活动的两种基本形 式和结构单位。形象思维与抽象思维相互相承, 密不可分。尤其是人类的抽象思维都是在语 言的基础上进行的, 而且是人类特有的, 在现实生活中应用最普遍的。思维时需要语言, 语 言和思维形影相随, 不可分离。语言能力的高低则自然与思维能力的强弱有着密不可分的联 系。在培养训练学生抽象思维的过程中, 语言中的语法教学显得尤为重要。语言符号是一个 组织有序的结构系统。语言内部的结构规律对基础语文教学具有多方面重要的指导意义。

例如, 语言学理论中的组合关系和聚合关系是语言结构的根本关系。语法的组合规则和 聚合规则就在这两种基本的结构关系之中, 从而构成了一种语言的语法规则。从本质上讲, 语法是人类抽象思维的产物。而语言学家则用数学符号、公式、规则等多种表达方式来描述 语法现象。对青少年进行基础语言教育, 其实就是对其思维优化培养, 这是发展智能最重要、 最基本的手段。而基础语文教学是语言教育的基本活动, 是语言教学与言语教学相结合的一 种实践活动。尤其是言语教学, 能让学生逐渐地在母语感性认识的基础上进而上升为理性认 识, 同时也是我们从小学到中学培养学生智能的一项最基本、最重要的活动。教育心理学理 论告诉我们:学生必须经由自己的心智活动才能对知识客体产生主观表征,才能对知识客体进 行加工和改造,才能将新知识纳入自己原有的知识结构中去。心智活动实质上就是“在人脑内 部,借助内部言语, 以简缩的形式,对事物的主观表征进行加工、改造的过程。”[3](第220页)所以 可以得出: 学生的心智活动与其思维、内部言语是水乳交融在一起的。人在思维时靠的是内 部言语, 内部言语是一种自己思考问题的言语活动。当然, 内部言语则需要通过外部言语来展 现。而且, 内部言语和外部言语是可以互相转化的。学生只有不断地通过这两种活动的实践, 才能更快地提升智能。这样看来,语文教学过程中绝对离不开内部言语和外部言语这两种言语 形式, 而这又是语言学理论的基本问题。

由此可见, 基础语文教学的一个十分重要的任务就是通过语言来训练学生的思维能力。 一个语文教师如果能深入研究并充分运用语言学知识理论, 并在其教学实践中不断探索、培 育学生合乎法则的言语心智活动和技能,自觉适时适度地诱发、指导和调控学生言语行为中两 种言语的互相转化, 这无疑对促进青少年学生智力的发展十分有利。 


\section{2 语言学理论在语文教学实践中对培养学生的分析语言现象与语境辩证关系具有十分重要 的作用}

语言现象纷繁复杂, 但在语言现象背后却隐含着许多内在的语言规律, 尤其是要结合特 定语境去分析研究。语境是语言学基础理论研究的一个重要方面, 它在语言研究和教学中起 着十分重要的作用。语境就是语言单位出现的环境。一般分为上下文语境和情景语境 (又叫 社会现实语境）两大类。“上下文”是指在一段话或一篇文章中凡出现在某语言单位之前或之 后的词、句都是该语言单位的上文或下文。上下文语境是就语言内部来说的。情景语境是指 说话时所涉及的人物、背景、时地、社会环境以及说听双方的辅助性交际手段（包括表情、 姿态、手势等非语言因素)。[4](第243页)情景语境是就语言外部来说的。由于语言总是在一 定的语言环境使用, 因此分析语言现象必须把它和它所依赖的语境结合起来, 离开特定的语 境把一个语言片段孤立起来分析研究, 这样往往会产生歧义, 难以确立这个语言片段的结构 和意义。语境是一切语文活动的言语环境的总和。任何语文活动都会受到语境因素的影响和 制约。特别是在理解词义和语义时要根据其所出现的语境来分析。作为人类思维与交际工具 的语言, 其词义具有概括性和确定性, 因此一个词往往有多种意义, 并为社会所约定, 这是 静态意义。但是, 词义还有一定的模糊性和民族性, 特别是虚词虽然没有实质意义, 但在文 言中通过相应的语法意义来表达词义, 这是其动态意义。词语只有在它进入具体的语境之后, 才能将静态义和动态义结合起来, 体现其意义的单一性和鲜明性, 这样我们才能更为具体的 理解词语所表达的生动含义。在语文教学过程中, 学生对词义的掌握是由少到多的。因此, 学生在学习中易受一些负迁移的影响, 生硬地理解已知该词的意义, 这样就造成了词义理解 上的错误。

因此, 在基础语文教学中, 教师不仅要有意识地运用语言学理论培养学生的语义分析能 力, 更应该考察其在具体语境中所表达的动态意义。只有这样, 学生才能真正把握语句中的 实际含义。

\section{3 语言学理论在语文教学实践中对提高学生的文学欣赏水平具有十分重要的作用}

“语文”在《现代汉语词典》释义有二：一指“语言和文字”，另一指“语言和文学”。[5](第 1601)然而, 目前学界对“语文”所指仍有分歧。多数认为: “基础语文”应包括“语言与文字”、“语 言与文学”的双重含义。[6]从语文教学实际看, 我们完全赞同其双重含义。既然语文教学具有 双重含义, 那么就产生了一个问题: 这双重含义之间究竟有何关系? 我们认为, 其关系就如 建筑材料与建筑物之间的关系, 而关键还是要看建筑设计师如何运用这些材料来打造独具特 性的建筑物。

学生语言能力的高低在于其语言材料是否丰富, 然而语言运用中的词语是有限、也是有 序的, 而文学作品中的语言材料却是无序的, 这是因为文学作品是一种语言艺术, 体现的是 作者的思想情感。文学作品教学是基础语文教学中的核心部分, 语言与文字的教学贯穿于其 中。正因为如此, 语文教材才以一篇篇言语作品为主要内容。语文教师在言语作品教学 (即 阅读教学) 中对语言知识的内化、转化最直接地体现在对文本解读的教学上。进行文学作品 解读教学时, 语文教师如能有意识地以相关语言学理论作指导, 分析作品的语言现象, 认识 作品的语言特点, 欣赏作品的语言艺术, 并从文本的语言层面、形象层面深入到文本的意蕴 层面, 这就能感受特殊的言语形式在言语内容上所表达的独特意味。[7]众所周知, 语言是文 学作品的外在形式, 是构成作品的媒介、符号, 同时也是作品沟通作者与读者的中介、桥梁。 语言是以文字为载体的, 文字又是形音义结合一体的符号, 语言文字的音、义则是构成文学 作品艺术的第一要素。所以, 从语言层面欣赏文学作品这一艺术, 我们应从语言文字的音、 义入手, 而不是逐字地去肢解这一艺术作品。诚如是, 我们才能真正而深刻地领悟作品整体 的思想内容和艺术美感。汉语是世界上最美的语言之一, 它具有音韵美与节奏美。无论吟诵 还是歌唱, 人们都会感受其音乐感和节奏美, 从而给人们传达作者的真实情感。例如, 李清 
照的《声声慢》以元音“i”加叠词“受受”“凄凄”“戚戚”“滴滴”的语言艺术,大大增添了凄清的情 绪。

欣赏文学艺术, 还应读懂语言的字词含义, 读懂语言的字词含义是读懂作品的前提条件。 文学作品的字词含义除其静态的本义外, 还有其动态的隐义（诸如引申义、双关义、象征义、 比喻义之类），此外还含有丰富的情感信息和复杂的生命韵味。例如，宋祁《玉楼春·春景》 中的“红杏枝头春意闹”的“闹”字，此处显然是其引申义，它有声有色，不仅写出了红杏众多、 纷繁的景象，还把生机勃勃的大好春光祄托出来了。王国维在《人间词话》中说：“着一“闹” 字而境界全出。”[]所以该词饱含了作者丰富的思想情感。此外, 还要注重作品的修辞效果, 揣摩作品的关键词语，思考作品的超常搭配，等等。

\section{3 语言学理论与语文教学实现成功对接的广阔前景}

\section{1 语文教学应对接语音学理论}

“语文”这一名称是新中国成立时开始使用的。之前有两种称谓, 小学谓之“国语”, 中学 以上谓之“国文”。至今港澳台及海外地区仍用前一称名。每人从幼儿开始就学习语言, 此时 主要受教于父母和周边的人, 通过模仿他们的语音来进行言语习得。因此, 在幼儿言语及小 学语文教学中, 我们应该充分发挥语音教学的作用。但遗憾的是, 如今的基础语文教学未能 达到理想的效果。语音教学大多仅停留在生字注音和朗读课文上。而中学连朗读也基本上没 有了, 仅在早自习让学生自由读二十分钟左右, 根本没有朗读训练, 更不用说运用语音学理 论来指导了。我们知道, 语音具有表意功能, 是一种特殊的声音。基础语文的语音教学先要 处理的是声、韵、调的教学。就声母教学而言, 从我们收回的 152 份调查问卷可以看出, 许多 小学教师仍然是按 $b 、 p 、 m 、 f 、 d \ldots .$. 这样的顺序来教学。在舌尖前音 $z 、 c 、 s$ 与舌尖后音 $z h$ 、 $\mathrm{ch} 、 \mathrm{sh} 、 \mathrm{r}$ 的教学上, 他们教学生的发音是: $\mathrm{z} 、 \mathrm{c} 、 \mathrm{~s}$ 是平舌发音, $\mathrm{zh} 、 \mathrm{ch} 、 \mathrm{sh} 、 \mathrm{r}$ 是尧舌发音, 其实这种方法是错误的。相对于舌尖前音来说, 舌尖后音是舌头往后缩而非翘舌所发出的音。 韵母教学可以由简到繁, 从单元音韵母到复合韵母进行教学, 主要教学生掌握元音发音这一 基本原理, 学会自主拼读。声调教学上, 普通话声调主要学习赵元任“五度标记法”, 让学生 能直观地了解其调值。小学拼音教学主要通过一些有趣的情景以及口耳传授引发学生的兴趣, 并从中渗透一些语音学基础知识, 让学生正确拼读书写声韵母及音节, 同时认识轻声、双音 节及多音节连续变调等常见的音变现象, 并能在短句的语调与声调关系的不同表达中领悟句 意的细微差别。如“这是你的”在生活中常用, 书面语有两种表达方法:

(1) 这是你的? (2) 这是你的。

（1）是个疑问句，读时句末音节读得大声一点，这样声调的调值就会升高，所以疑问句 为升调; 而 (2) 是个陈述句, 句末音节读的声小了一点, 调值下降了, 所以表肯定的陈述句 为降调。这就涉及到语音学理论的基本句调问题。这是就一个简短的话语而言, 而随着年级 的逐步上升, 语文教学以文本为基础, 将各个教学内容贯穿其中, 这时就需要学会如何有感 情的朗读。因此, 这就要求教师自身要储备停顿、节奏、重音、语调等语音节律知识, 并且 有意识地引导学生掌握这一基本技能。

\section{2 语文教学应对接词汇学理论}

在语言三要素中, 词汇作为语言的建筑材料是最丰富、最活跃的, 也是最重要的。虽然 词汇具有如此特征，但它有着严密的系统性。

首先, 所有的词语都是由基本语素构成的, 这些语素大多是词根, 这是词汇外在的根本 形式。例如, 汉语中的语素“水”, 可以构成数百个含“水”的词语, 虽然其形式不一，但却万 变不离“水”的基本含义。 
其次, 词语是各语素遵循一定规则构成的，如合成词复合式的基本构词类型—并列式、 偏正式、补充式、动宾式、主谓式。

再次，那就是词义，这是词汇内核的根本形式。语言中每个词都有其特定的意义，词义 是有其客观性、概括性、模糊性、民族性等特点的，同时还有其理性义与色彩义之辨。一个 词从理性意义上讲一般有两种以上的意义。这时，我们就要从词义分析的角度，根据我们对 该词所掌握的意义并结合相应的语境, 来正确区分语境中所表示的是基本义还是转义。例如, “深”虽有八个义项，但仅有一个基本义：“从上到下的距离”，其余七个义项都跟“距离大”这 个意义有关。另外, 在语言运用过程中, 学生所说的与所写的句子从理性义上是通的, 但为 何又有一定的错误呢? 这可能是色彩义上出现了一定的偏差。如下例就将词的贬义错用为袋 义:

司机张师傅冒着生命危险解救乘客的事迹，一经新闻媒体报道，就被传得满城风雨，感 动了无

数市民。(2010 年江苏高考卷)

“满城风雨”常用于指坏的、不好的事到处传播，到处议论，显然带有贬义，而句中是指 英雄事迹，表达颂扬之义，因此情感意义不符。

文言词汇的积累也是基础语文教学的一大目标，高中阶段则要求学生必须建立完整的文 言词汇体系。《全日制高中语文教学大纲》附录一列举了常见的文言实词150个, 虚词15个。 这些实词均属基本词汇范围之内的，对于这些实词的讲授，必须运用词汇学知识理论:

首先, 文言中部分词语沿用至今, 其义已经发生了变化。学习这些词语, 就应考察古今 义的异同，掌握其词义扩大、缩小、转移、襄贬义转换等内容。例如:

河内凶，则移其民于河东。(高中语文《寡人之于国也》)

“河”古代是专有名词，专指黄河，而今泛指一切大小河流，其词义范围扩大了。

开我东阁门，坐我西阁床。（初中语文《木兰诗》）

“床”古代兼指坐具和卧具, 而现在仅指卧具, 其词义范围显然缩小了。

哙拜谢,起,立而饮之。（高中语文《鸿门宴》）

“谢”的本义, 《说文》释之“辞去也”。而例中的“谢”则是“感谢”义，与其本义已无类属关 系, 词义发生转移了。

\section{将军者，国之爪牙也。（《汉书·李广传》）}

“爪牙”古指“武臣”或“得力助手”，而今义为“帮凶”“走狗”，已由襄义转为贬义了。又如“锻 炼”，古指“玩弄法律诬陷人”，今已由贬义变为襄义了，如“思想锻炼”“锻炼身体”等。

其次，文言词语大多以单音词为主，其所包含的义项比现代汉语双音词多，因此一词多 义是其普遍现象。文言一词多义现象对学生的阅读造成了一定的困难, 这也成为词汇教学的 主要内容。

再次，文言词语教学还涉及通假问题。“通假”是以音同或音近的字代替本字的一种语言 现象。在文言词语教学中, 语文教师要引导学生分析通假现象, 而不是生搬硬套地机械记忆。

此外，还有文言虚词问题。文言虚词缺少一定的系统性，不像实词那样有明显的词汇意 义, 它更多的是表达语法意义。如何让学生掌握、积累文言虚词, 这是一个十分关键的问题。 所以, 对文言虚词的讲解, 语文教师可以先引导学生通过理清词的演变规律来把握。虚词是 由实词转变过来的, 只要学生把握了虚词的演变规律, 不仅知其然还知其所以然, 这就可以 避免死记硬背，也易分辨而不致混淆。如“之”是文言中用得最多的一个虚词。《说文》云: “之, 出也。”段注之曰: “引申之义为往。”因此“之”的本义为“出生、滋长”, 又引申为“往”或“到” 等义, 动词。例如:

尝与人佣耕, 辍学耕田之垄上。（初中语文《陈涉世家》）

由于“往”什么地方去, 与之相对的就是从什么地方来, 就是出发点, 因此出发点我们常 说“这”、“此”，因此“之”始引申为“这”、“此”意，具有代词的功能。例如： 


\section{闻之，欣然规往。（初中语文《桃花源记》）}

此外, “之”作助词不仅用之最广, 而且也最复杂, 其与本义无关, 显然为假借之用法。 例如:

（1）向读之不知, 惑之不解, 或师焉, 或不焉。（高中语文《师说》）

（2）蚓无爪牙之利，筋骨之强。（高中语文《劝学》）

（3）客之美我者, 欲有求于我也。（初中语文《邹忌讽齐王纳谏》）

（4）久之, 目似瞑, 意暇甚。（初中语文《狼》）

上述例子中, 或为宾语前置的助词, 如例 ( 1 ); 或作定语后置的助词, 如例（2）; 或位于主谓之间取消句子独立性, 如例（ 3 ）; 或无义仅起凑足音节的作用, 如例（4）, 等等。

\section{3 语文教学应对接语用学理论}

“语文课程与教学, 实质上是一种言语教育, 应当属于语用研究的范畴。语文教学离不开 语言的语音、语义、词汇和语法规则系统, 但是语文课程的根本目的不是培养学生去研究语 言, 而是培养学生具有理解和运用祖国语言文字去进行交际的言语能力。”[8]因此, 基础语文 教学实际上是语用教学。从 20 世纪 30 年代语用学概念的产生到成为一门独立的学科, 它为语 言学研究和语言教学提供了广阔的前景。

多年来, 语文教学以静态的语言单位作为主要的教学分析对象, 并且重在词语、句子的 书面意义上, 从片段的上下文来理解阐释, 而很少从整个语言使用行为的过程中去分析研究。 这样一来, 学生就不易理解教师的抽象讲授, 也难以灵活而娴熟地运用其语言理论知识。而 语用学则从整个语言使用行为过程中来考察词义或句义, 当然其中最主要的是通过语境对其 进行推理, 捕捉词义或句义背后说话人的语用意图及语用效应。人们对其语用意义作出解读, 就要涉及许多变量, 因为它以实际的言语交际活动为主, 更贴近实际活动中的语言生活。目 前, 语用学对各种不同类型的语用意义进行了一定的研究, 研究得较为深入的有: 指示现象、 会话隐涵、规约隐涵、预设等。[9]这些研究为我们深入解读文本的语用意义提供了有效的方 法。

传统语言学研究的最大单位是句子, 对句子功能的认识主要反映在句类（如陈述句、祈 使句、疑问句、感叹句) 的划分上。而语用学主张的则是言语行为, 这一理论进一步深化了 人们对语言功能的认识。言语行为理论给我们的启示是：语言表达功能远比上述四种功能大 得多。言语行为理论认为, 一个言说动词就是一个语言功能, 一种语言里言说动词是成百上 千的, 如抱怨、威胁、邀请、建议、宣布、通知等。塞尔把言语行为的类别分为阐述类、指 令类、承诺类、表态类和宣告类, 就是一个有益的尝试。如果我们在语文教学中能区分这些 不同的类型, 分别展开实境化的练习, 那么对提高学生的社会语用能力是大有禆益的。[10]

语用学以言语行为作其研究对象, 认为实施言语行为的单位不仅仅是一个个句子, 也可 以是局部语篇甚至整个语篇。它认为, 唯有一个语篇才能完整地实现一个语用意图, 因此语 用学开创了比句子更为复杂的语篇研究范式。所谓语篇, 通常是指一系列连续的话段或句子 构成的语言整体, 它是我们日常生活中语言的主要表现形式, 短者两三句, 长者洋洋万言。 每个语篇都有自己的构造规律, 通过语篇结构表现出来。[10]语篇结构理论其实是运用整体的 思想来研究言语行为。由于每个语篇都有主题, 从整体入手就容易把握语篇的主题和结构。 在语篇结构研究中, 语用学发展了一系列新的理论方法, 诸如宏观结构研究、衔接手段研究、 主题推进程序研究, 等等。这些语用研究的理论方法对于基础语文这种母语教学来说是非常 有用的, 因为文本的篇章感是语感的重要内容。根据我们的调查, 中学生的语法感知能力一 般没有问题, 而篇章感知能力则有待进一步加强。例如, 写作的问题主要不是句子的语法不 通, 而是篇章的文脉不畅, 其病因可以通过语篇结构教学来解释。 


\section{4 结束语}

社会在不断发展, 语言学理论随之也不断地丰富, 其应用领域越来越广泛, 而且正日益 发挥着重要作用, 展现出美好的发展前景。我们深信, 在这个新时代, 随着教育事业的大发 展, 语文教育改革也必将进一步深入, 语文教学实践不仅迫切需要语言学理论作指导, 而且 还会把语言学理论有机地融入于本学科的教学实践之中。诚如是, 语言学理论与语文教学实 践才是真正实现了成功对接, 其前景一定是美好而广阔的。

\section{致谢}

本文为中国江西省高等学校教学改革科研课题“高师语言学理论与基础语文教学实践问 题研究——关于如何加强高师语言学理论与基础语文教学实践对接问题的思考” (JXJG-13-2-4) 的阶段性成果之一。

\section{References:}

[1] Ye Feisheng, Xu Tongqiang. Linguistics Outline[M].BeiJing:Peking University Press,2010.

[2] Ni Baoyuan.Linguistics and Chinese education[M].Shanghai:Shanghai Education Press,2001.

[3] Feng Zhongliang.Structure - oriented teaching principle and practice(volume One)[M].BeiJing:Beijing Normal University Press, 1992.

[4] Huang Borong,Liao Xudong.modern Chinese(volume One)[M].BeiJing:Higher Education Press, 2017.

[5] Chinese Academy of Social Sciences Institute of language.modern Chinese dictionary[M].BeiJing:Commercial Press,2016.

[6] Su Baorong.Language theory research and language teaching[J].Journal of Hebei Normal University(Philosophy and Social Sciences Edition),2002(6).

[7] Gao Jing.Linguistic knowledge in Chinese teaching system[J].The language learning,2012(1).

[8] Han Xuepin.New perspective on language knowledge[J].The language learning,2004(5).

[9] Shen Jiaxuan.The boundary between pragmatics and semantics[J].Foreign language teaching and research,1990(2).

[10] Xu Mofan.Language Teaching Reform from the Perspective of Pragmatics[J].Language building,2015(19). 